\title{
Perceived Quality of Multimedia Educational Content: A Cognitive Style Approach
}

\author{
GHEORGHITA GHINEA* AND SHERRY Y. CHEN
}

School of Information Systems, Computing and Mathematics

Brunel University

Uxbridge, UB8 3PH, UK

\{George.Ghinea, Sherry.Chen\}@brunel.ac.uk

\begin{abstract}
Cognitive styles influence the way how humans process information, with previous research demonstrating that they have significant effects on student learning in multimedia environments. On the other hand, the perceptual quality of the human multimedia experience is a notoriously difficult to measure. In this paper, we report the results of an empirical study, which investigated the relationship between user cognitive styles and perceptual multimedia quality, in which users had the possibility to specify their desired Quality of Service settings - in terms of frame rates and color depth. Results show that whilst color choice is impacted by a participant's cognitive style, such Quality of Service parameters do not significantly affect perceived multimedia quality, and that users do not necessarily choose optimum presentation settings to enhance their perceived enjoyment and assimilation of multimedia informational content.
\end{abstract}

Key words: Cognitive Styles, Human Factors, Multimedia, Perceptual Quality, Quality of Service, Learning Style

\section{Introduction}

Multimedia technologies play an increasingly important role in education (Chou and Lin, 1998; Mayer, 2003; Schnotz and Lowe, 2003). However, whilst adaptive multimedia approaches in e-learning abound, very few of them (Oberlander and Gill, 2004; Gill, 2003) address perceptual quality issues. Research in this area has highlighted several key concerns, one of which deals with the effectiveness of multimedia presentations (Garrett and Callear, 2001). To this end, an important measure of the effectiveness of a multimedia presentation is the user experience captured in terms of enjoyment and information assimilation. Key is the issue of the quality of the multimedia presentation.

In a distributed multimedia environment, the issue of quality takes on a new dimension, as not only is it in the eye of the beholder, but is also affected by the underlying communication system. Accordingly, in our perspective, quality has two main facets: of service and of perception. The former, Quality of Service (QoS), illustrates the technical side of computer networking and represents the performance properties that the underlying network is able to provide. The latter, Quality of Perception (QoP), characterizes the perceptual experience of the user when interacting with multimedia applications. Whilst the quality delivered by communication networks has traditionally been measured using QoS metrics, we believe that, as learners are 'consumers' of multimedia educational applications, it is their opinions of and experiences with the quality of multimedia material that ultimately measure the success (or, indeed, failure) of such applications to successfully deliver edutainment (i.e. combined educational and entertainment) material. Moreover, when this delivery is done over Wide Area Networks such as the World Wide Web ('the Web'), transmission of multimedia data has to accommodate not only users' subjective 
preferences, but also fluctuating networking environments.

The question also arises of whether distributed multimedia presentations can accommodate individual preferences. Previous research has indicated that users with different characteristics have different perceptions of multimedia presentations (Chen and Angelides, 2003). In particular, different cognitive style groups benefit from different types of multimedia presentation. Empirical examination of cognitive style impact is eminently necessary, because such an endeavor has the potential to offer concrete prescriptions for developing user-centered systems that can match the particular needs of each cognitive style group.

In this paper, we present the results of an empirical study that explored the relationship between user cognitive styles and QoP, when multimedia is presented with varying levels of QoS parameters. The results of this study can help designers to understand how to choose appropriate multimedia presentation parameters to match the preferences of users with different cognitive styles, and in so doing, make better use of scarce networking resources such as bandwidth. Accordingly, this paper is structured as follows: Section 2 presents a review of issues related to QoP and cognitive styles research; the methodological design of our empirical work is then detailed in Section 3, while results are described and discussed in Section 4. Lastly, conclusions are drawn and possibilities for future work identified in Section 5.

\section{Background}

\subsection{Perceptual Multimedia Quality}

Communication networking research primarily treats issues of quality from a technical perspective - to this end, QoS parameters are specified, managed and adapted. However, such parameters do the user no favors, for they do not convey specific needs of applications, such as the influence of clip content and informational load on the user multimedia experience, nor do they encapsulate user media preferences (e.g. video over text, animation over graphics) or characteristics.

It comes as no surprise therefore that relatively little has been reported on the relationship between the network provided QoS and the satisfaction and perception of the user. Indeed, the underlying assumption of studies, which have explored user perception of multimedia in the educational psychology and Human-Computer Interaction fields (see, for example, Garrand, 1997; Hapeshi and Jones, 1992; Mayer, 2003; Oberlander and Gill, 2004; Reeves and Nass, 2000), has been that the underlying network is able to provide an optimum QoS and, therefore, an excellent quality of multimedia presentation - an optimistic premise to say the least. Moreover, this assumption also underlies the prevailing research underpinning multimedia learning. We know, for instance, how humans process and retain information (Gross, 1994), how human retention varies with the number of sources from which the information originates (Hapeshi and Jones, 1992; Lewalter, 2003), the individual contribution of media effects to learning (De Westelinck et al, forthcoming; Lowe, 2003; Mayer, 2003), the perceptual impact of lexical constructs (Bradac et al., 1988), as well as how to induce humans to think in a certain way (Cialdini, 2000; Xia and Lee, 2000). However, as any user who has viewed multimedia content over the Web will testify, such transmissions are rarely free from quality issues - media losses, errors and degradations are all factors which impact upon the user multimedia experience and which must be explored if human factors are to be truly integrated in distributed multimedia learning systems.

That the delivered QoS impacts upon perceived multimedia quality in distributed systems is generally accepted - however, previous work examining the influence of varying QoS on user perceptions of quality has almost totally neglected multimedia's edutainment duality. In this respect, research has concentrated primarily on the perceived entertainment value of presentations displayed with varying QoS parameters. Accordingly, Apteker et al. (1995) examined the influence that varying frame rates have on user 
satisfaction with multimedia quality, whilst Steinmetz (1996) presented the perceived effect of synchronization skews between media. In related work, Wijesekera et al. (1999) investigated the impact of media loss on users, while the establishment of metrics for subjective assessment of teleconferencing applications was explored by Watson and Sasse (1997). Indeed, the correlation between a user's subjective ratings of differing-quality multimedia presentations and physiological indicators has been studied in Wilson and Sasse (2000), whilst perception of personalities in computer mediated communication and, implicitly, in the presentation of multimedia content was explored by Gill (2003).

To summarize, previous studies exploring perceptual distributed multimedia quality can be characterized by three main observations:

- they have highlighted the potential for significant resource savings to be made if perceptual considerations are integrated in the transmission of multimedia content (of especial importance in bandwidth constrained environments such as Webbased distance learning systems);

- they have failed to explore the influence of user choice, when there is a cost associated to it, on perceptual multimedia quality, and

- they have concentrated almost exclusively on the entertainment dimension of multimedia (ignoring the informational/ educational aspect).

Whilst the former two observations provide the main drivers behind our work, the latter is the motivation for the use in our research of the QoP metric (Ghinea and Thomas, 1998). This represents, to the best of our knowledge, the only metric for perceptual quality evaluation which takes into account multimedia's edutainment characteristic. With it users are asked to indicate, on a scale of $1-6$, how much they enjoyed the multimedia presentation (with scores of 1 and 6 respectively representing "no" and, "absolute" user satisfaction), while their knowledge of the informational component is examined via a series of 10 questions, and expressed as a percentage measure reflecting the proportion of correct answers received.

We believe that a measure such as QoP, which specifically targets the edutainment aspect of multimedia, will have more meaning for a typical user than QoS metrics, and, in this paper, we explore the impact of individual differences, as given by the user's cognitive style (an individual's characteristic and consistent approach to organising and processing information (Riding and Grimley, 1999)) on QoP

\subsection{Cognitive Styles}

Among a variety of cognitive styles, previous research indicated that the dimensions of Field Dependence/-Independence have significant effects on individuals' preferences to multimedia information systems, because it reflects how well a user is able to restructure information based on the use of salient cues and field arrangement (Riding, 1991; Weller, et al., 1994). Based on this taxonomy, users are classified as Field Independent, Intermediate, or Field Dependent, with the main differences being found between Field Independent and Field Dependent users. Table 1 presents the defining characteristics of Field Dependent and Field Independent individuals, with persons who possess combined characteristics of both Field Independence and Field Dependence being considered as Intermediate (Riding, 1991).

\section{\{Table 1 Here $\}$}

Several studies have suggested that Field Independent individuals could particularly benefit from the control of media choice. Thus, four courseware versions were produced in (Chuang, 1999): animation+text, animation+voice, animation+text+voice, and free choice. The result showed that Field Independent subjects in the animation+text+voice group or in the free choice group scored significantly higher than those did in the other two groups. No significant presentation effect was found for the Field 
Dependent subjects. Conversely, Lee (1994) indicated that auditory cues are important to Field Dependent learners. In addition to the aforementioned educational settings, a number of studies have examined the effect of Field Independence on online searching (e.g. Ford et al., 1994; Ford et al., 2001).

Whilst the majority of research examining the influence of cognitive styles in multimedia learning environments has focused on navigation styles (Avitabile, 1998; Beacham, et al., 2002) and presentation modes (Chou and Lin, 1998; Ford and Chen, 2000) there is a distinct lack of research examining the relationships between the provision of networklevel QoS and the requirements of different cognitive style groups in distributed multimedia environments. Exploration of these issues is, in fact, paramount, because such an evaluation can shed light on efficient use of network resources and could potentially result in better end-to-end system performance, which in turn has a direct positive impact on the user experience of multimedia.

\section{Methodology Design}

\subsection{Participants}

71 students from an UK university volunteered to take part in the study. A request was bulk email-ed to undergraduate students inviting them to participate in an experiment which, participants were told, should last no longer than an hour, would involve watching multimedia presentations and soliciting responses and opinions based on their content.

When we applied Riding's Cognitive Style Analysis (see Section 3.3), participants turned out to be quite evenly distributed in terms of cognitive styles, including 23 Field Independent users, 25 Intermediate users, and 23 Field Dependent users. Moreover, as Table 2 depicts, participant breakdown according to gender was also quite evenly matched. All users tested were inexperienced in the content domain of the multimedia video clips visualized as part of our experiments, which will be described next.
\{Table 2 Here

\subsection{Video Clips}

A total of 12 video clips were used in our study. These 12 clips were between 30-44 seconds long and digitized in MPEG-1 format. The subject matter they portrayed was varied (as detailed in Figures 1 and 2) and taken from selected television programmes, thereby reflecting edutainment sources that average users might encounter in their everyday lives. Also varied was the dynamism of the clips (i.e., the rate of change between the frames of the clip), which ranged from a relatively static news clip to a highly dynamic Space movie, the degree of dynamism of a clip being established through user tests, as detailed in Ghinea and Thomas (1998), where full descriptions of the clip content can also be found.

\{Figures 1, 2 Here $\}$

\subsection{Cognitive Styles Analysis}

The cognitive style dimension investigated in this study was the level of Field Dependence. A number of instruments have been developed to measure Field Dependence, including the Group Embedded Figures Test (GEFT) by Witkin et al. (1971) and the Cognitive Styles Analysis (CSA) by Riding (1991). The main advantage of the CSA over the GEFT is that Field Dependent competence is positively measured rather than being inferred from poor Field Independent capability (Ford and Chen, 2001). In addition, the CSA offers computerized administration and scoring. Therefore, the CSA was selected as the measurement instrument for Field Dependence in this study.

The CSA includes two sub-tests (Figure 3). The first presents items containing pairs of complex geometrical figures that the individual is required to judge as either the same or different. The second presents items each comprising a simple geometrical shape, such as a square or a triangle, and a complex geometrical figure, as in the GEFT, and the 
individual is asked to indicate whether or not the simple shape is contained in a complex one by pressing one of two marked response keys (Riding and Grimley, 1999). These two subtests have different purposes. The first sub-test is a task requiring Field Dependent capacity. Conversely, the second sub-test requires the disembedding capacity associated with Field Independence.

\{Figure 3 Here

The CSA measures what Riding and Sadler-Smith (1992) refer to as a Wholist/Analytic (WA) dimension, noting that this is equivalent to Field Dependence/ Independence. As Witkin et al. (1971) argued, a Field Independent individual is capable of a more analytical cognitive function than a Field Dependent individual, who uses a more global approach. Riding's (1991) recommendations are that scores below 1.03 denote Field Dependent individuals; scores of 1.36 and above denote Field Independent individuals; students scoring between 1.03 and 1.35 are classed as Intermediate. In this study, categorisations were based on these recommendations.

\subsection{Quality of Perception}

User perceptual multimedia quality was assessed using the Quality of Perception measure. Consistent with its ability to poll edutainment quality, this metric has two components - one which measures the ability of users to understand and assimilate the informational content of clips, denoted by QoPIA, with the other, QoP-LOE, measuring the subjective level of enjoyment with the multimedia quality. The first is expressed as a percentage measure reflecting the proportion of answers correctly given to a series of 10 clipspecific questions that users had to answer after watching each clip in our selection, whilst the second is expressed on a Likert scale of 1-6 (with scores of 1 and 6 representing the worst and, respectively, best perceived qualities possible).

\subsection{Procedure}

Due to a small sample size, a within subject design was selected for experiment design (Reips, 2000). In this way, each participant chooses his/her desired presentation settings, and one can thus examine how cognitive styles influence their choices. To help them choose suitable settings, participants were given an overview of the process that was to proceed in the opening screen, (Figure 4). The following screens were those of the computer-presented CSA to determine the individual participant's cognitive style. Once this was done, users moved onto the 'Test Movie' Screen, where they were given a chance to become familiar with varying levels of QoS delivery, by visualizing appropriately parameterized probes of multimedia material. Given their importance in the utilization of bandwidth (a scarce resource in multimedia systems), and in keeping with previous work done in this respect (Apteker at al.; 1995, Ghinea and Thomas, 1998; Wijeskera et al., 1999), two QoS parameters were considered in our experiment frame rate and color depth. Accordingly, users could select multimedia probes which exemplified playback at three different frame rates $(25,15$ and 5 frames per second) and two different color displays (24-bit color and grayscale, respectively).

\section{\{Figure 4 Here}

Having become familiar with the varying levels of QoS delivery, users were then requested to select their preferred choice of QoS delivery for the 12 video clips of our experiment. Users were told that their particular choice would impact upon their final scores, in an inversely proportional relationship to their specified quality levels. The final stage of the experiments involved participants watching the twelve video clips. After each video clip was seen, participants answered the QoP-IA questions and indicated their QoP-S ratings for the respective clip. In order to counteract any order effects, the order in which clips were visualized was varied randomly for each participant. 


\subsection{Data analysis}

In this study, the independent variables include the participants' cognitive styles, as well as clip categories, and degree of clip dynamism (Table 3). The dependent variables were the two components of Quality of Perception, QoP-IA and QoP-LOE.

\section{\{Table 3 Here $\}$}

Data were analyzed with the Statistical Package for the Social Sciences (SPSS) for Windows version (release 9.0). An ANalysis Of VAriance (ANOVA), suitable to test the significant differences of three or more categories (Stephen and Hornby, 1997) was applied to analyze the participants' responses. A significance level of $p<0.05$ was adopted for the study.

Since in real-life quality often comes with a hefty price tag, in our experiments we scaled participants' QoP-IA scores through a user preference coefficient:

$$
\text { preference coefficient }=5 *(\text { color } * f p s)^{-1}
$$

The main role of the preference coefficient was to incorporate the impact of user QoS choices on their QoP-IA score (the higher the frame rate and the better the color quality requested, the lower the scaling coefficient) and, as opposed to previous studies in the area (Apteker et al., 1995; Ghinea and Chen, 2003; Watson and Sasse, 1997; Wijeskera et al., 1999), which have exclusively ignored the issue, also reflects the extent to which personal entertainment considerations ('I want the highest possible multimedia display quality...') are balanced by informational ones ('...but do I really need it if information capture is also my aim?') in the context of multimedia edutainment.

Accordingly, in our experiments color $=1$ for grayscale presentations and 3 for 24-bit color presentations (representing the number of bytes needed to encode color information using each of the two schemes), while fps is the numerical value of the desired frame rate, expressed in frames per second. The preference coefficient thus also gives a relative indication of bandwidth resources required by the chosen multimedia presentation (a value of 1 corresponding to a low bandwidth, grayscale, $5 \mathrm{fps}$, presentation, with the lowest value of $0.0(6)$ corresponding to a full quality $25 \mathrm{fps}$, color presentation).

\section{Results and Discussions}

\subsection{The influence of cognitive style on $Q o P$}

Our results show that there were no significant QoP differences - in respect of both information understanding and assimilation, as well as user satisfaction - across cognitive styles, irrespective of the particular QoS levels chosen by the participants for the clips (Figure 5). At first glance, this is a surprising result for our study shows that even normalised (through equation (1)), QoP-IA scores are not dependent upon cognitive styles, and that the user perceptual multimedia experience is not affected by the underlying, chosen, QoS parameters such as frame rate and color depth.

This finding has two implications, for not only does it reconfirm previous research (Apteker et al., 1995; Ghinea and Thomas, 1998) which showed that the QoS of a multimedia presentation can be significantly degraded without a proportional decrease in multimedia perceptual quality, but it also shows that this observation holds true even when users are allowed to choose their own QoS. Moreover, our findings complement those of previous research (Huang, 2003), which found that cognitive styles of users do not affect their navigation efficiency when browsing through multimedia content on the Web.

$\{$ Figure 5 Here $\}$

This is only part of the story, however, for, as detailed in previous work (Ghinea and Chen, 2003), neither is non-normalised QoP-IA dependent on user cognitive style, nor does it depend on the same QoS parameters varied in our study. Thus, our work suggests that users, when categorized along cognitive style 
dimensions, are not only generally unaware of quality differences, but also that they may have difficulty in choosing optimum quality levels which would lead to enhanced user QoP.

\subsection{Color choice and clip dynamism}

An interesting trend highlighted during data analysis was that of color depth selection by the different cognitive styles. Accordingly, when an Analysis of Variance was performed, it was found $\mathrm{t}$ hat for the Commercial $(\mathrm{p}=0.050)$, Documentary $(\mathrm{p}=0.028)$, Pop Music $(\mathrm{p}=0.015)$ and Cooking $(p=0.002)$ video clips, the particular choice of full color or grayscale was significantly influenced by the participants' particular cognitive style (Table 4). Moreover, what is interesting is that all of these clips are of medium dynamism, our findings suggesting that for clips at the end of the dynamism spectrum cognitive styles do not influence color choice selection (Figure 6). Moreover, for all twelve clips considered in our sample, our analysis revealed that frame rate choices are not influenced by an individual's cognitive style.

\section{\{Table 4 Here $\}$}

A further revealing feature of our results was that most Intermediate participants preferred to view (with one exception, the Space clip) color clips for all content visualized. This contrasts with the other two cognitive style groups, in which the particular preference for grayscale or color delivery was much more evenly balanced (Figure 6). This finding suggests that Intermediate participants had consistent color preferences, regardless of the subject content, because they tended to take versatile approaches, as reported in (Ghinea and Chen, 2003).

\{Figure 6 Here

\subsection{Clip Content and QoP}

We also undertook an ANOVA to explore the effect of clip content, as expressed through the particular dynamism of a clip, on QoP (Table 5). The results highlighted that clip dynamism, has, for all users, a significant effect on QoP-IA - irrespective of a particular user's cognitive style. Accordingly, the highest QoP-IA scores were obtained for clips with medium dynamism, for all categories of users. At the other end of the spectrum, highly dynamic clips were shown to have a detrimental impact on QoP-IA, with users obtaining low QoP-IA scores for clips in this particular class. Whilst we believe that the explanation behind these results might lie in the fact that the rapid temporal changes of dynamic clips make it difficult for the user to focus on their informational content, the observation that users do not obtain the highest QoP-IA scores for static clips might be explained by their failure to capture and focus user attention. Lastly, it is worthwhile to remark that in the clips with strong and medium dynamism, Field Dependent users performed worse than the other two groups while Field Dependent users had a (slightly) better performance than Field Independent users in the clips with weak dynamism. This may be due to the fact that weak dynamism clips provide less confusion and may thus be suitable for Field Dependent users who have difficulties in dealing with complexity (Witkin, et al., 1977). It also suggests that matching users' preferences can enhance their performance (Ford and Chen, 2001).

\section{\{Table 5 Here $\}$}

QoP-LOE seems to follow a similar pattern to that of the color choices. Thus, most of the clips which were identified as being of medium dynamism had a significant impact on QoPLOE, depending on a particular user's cognitive style (Figure 7): Commercial $(\mathrm{F}=4.636, \mathrm{p}=$ $.013)$, Cooking $(\mathrm{F}=3.923, \mathrm{p}=.024)$, Animation $(\mathrm{F}=6.032, \mathrm{p}=.004)$, Pop Music $(\mathrm{F}=13.495, \mathrm{p}=$ $.000)$. Field Independent users get more enjoyment than other cognitive style groups for clips which are preponderantly of medium dynamism. Again, for clips with extreme forms of dynamism, enjoyment is not impacted upon by cognitive styles. 
One would expect that if users were allowed to choose their quality settings, they would generally be happy with the resulting viewing quality of the multimedia clips displayed. This is not the case with our results, where QoP-LOE scores occupied quite a wide spectrum. However, one must remember that viewers did not watch the video clips for entertainment purposes alone, and that, in our experiments, they were also examined on the informational content of the multimedia video clips. It would thus seem that the particular task at hand does impact on user QoP-LOE, in spite of users having viewed the particular clips at quality levels chosen by them.

\section{\{Figure 7 Here $\}$}

\section{Conclusions}

In this paper, we have presented the results of an empirical study which examined the influence of user cognitive styles and QoS preferences (in terms of presentation frame rate and color depth choices) on perceptual multimedia quality. Results accordingly suggest that frame rate variations and color choices do not, irrespective of cognitive style, significantly impact upon perceptual multimedia quality, a finding of particular interest in bandwidthconstrained environments. Moreover, Intermediate users were shown to have largely versatile color choices.

Nonetheless, our results indicate that the perception of multimedia quality by the end user does not seem to be affected by the underlying QoS with which that multimedia was delivered, nor by the particular cognitive style of the user. This has a two-fold implication the first is that in bandwidth constrained environments, user QoP is largely unaffected by the presentation quality. Therefore, this offers both scope and promise. Scope - for more efficient utilization of resources, since playing back a multimedia clip at less than full blown quality is likely not to be noticed by users, or, even if it is, it is not likely to significantly affect his/her perceptual quality experience. Promise for it seems to suggest that personalization, though undoubtedly an important issue in the advanced communication systems of today and of the future, need not necessarily consider the cognitive style dimension in the tailoring of multimedia quality.

The second implication of our study is that our findings show that, even when given choice, the choices made by users do not necessarily lead to a better perceptual experience, if one considers informational assimilation as part of this. However, whether it is psychologically more important for users to be given the opportunity to make decisions regarding quality preferences is still an open question as far as multimedia is concerned.

Lastly, although we recognize that we have only examined a reduced subset of multimedia content and that, for instance, more clips with dynamic features could have been included in our sample, we cannot ignore the relatively low information assimilation scores associated with a subset of the multimedia content of our experiments. That this happened in spite of users being told that the information assimilation aspect of their experience was going to be evaluated, leads us to conclude that further work should also involve the influence of task and content on the subjective multimedia experience. This is a direction we are currently engaged in and will provide a valuable cornerstone in our efforts to build integrated multimedia communication systems .information. In Figure 6 , the results are presented in alphabetical order and there is an alphabetical index to help users effectively to locate a result of interest.

\section{References}

1. Apteker, R.T., Fisher, J.A., Kisimov, V.S. and Neishlos, H. (1995) Video Acceptability and Frame Rate. IEEE Multimedia. 2(3), 32-40.

2. Avitabile, J. (1998) Interaction of Presentation Mode and Learning Style in Computer Science. Proceedings of the National Educating Computing Conference. San Diego, USA, June 22-24, 1998.

3. Beacham, N. A., Elliott, A. C., Alty, J. L., and AlSharrah, A. (2002) Media Combinations and Learning Styles: A Dual Coding Approach. Proceedings of EDMEDIA 2002 World Conference on Educational Multimedia, Hypermedia \& Telecommunications. 
4. Bradac, J., Mulac, A., and House, A. (1988). Lexical diversity and magnitude of convergent versus divergent style shifting: Perceptual and evaluatory consequences. Language and Communication, 8, 213-228.

5. Chen, S. Y. (2002) A Cognitive Model for Non-linear Learning in Hypermedia Programmes. British Journal of Educational Technology. 33(4), 453-464.

6. Chen, S. Y. and Angelides, M. C. (2003) Customisation of Internet multimedia information systems design through user modelling, in: Architectural Issues of Web-Enabled Electronic Business , Shi Nansi Ed., Idea Group Publishing, 241-255.

7. Chen, S. Y. and Macredie, R. D. (2004) Cognitive Modeling of Student Learning in Web-based Instructional Programs. International Journal of Human-Computer Interaction. 17(3), 375-402.

8. Chou, C. and Lin, H. (1998) The effect of navigation map types and cognitive styles on learners' performance in a computer-networked hypertext learning system. Journal of Educational Multimedia and Hypermedia. 7(2/3), 151-176.

9. Chuang, Y-R. (1999) Teaching in a Multimedia Computer Environment: A study of effects of learning style, gender, and math achievement. Available: http://imej.wfu.edu./articles/1999/1/10/index/asp [21/12/2001]

10. Cialdini, R.B. (2000) Influence: Science and Practice, Addison-Wesley, Reading, Ma.

11. De Westelinck, K., Valcke, M., De Craene, B. and Kirschner, P. (forthcoming) Multimedia learning in social sciences: limitations of external graphical representations, Computers in Human Behavior, [24/11/2004].

12. Ford, N. and Chen, S. Y. (2000) Individual differences, hypermedia navigation and learning: an empirical study. Journal of Educational Multimedia and Hypermedia. 9(4), 281-312.

13. Ford, N., Miller, D. and Moss, N. (2001) The Role of Individual Differences in Internet Searching: An Empirical Study. Journal of the American Society for Information Science and Technology, 52(12), 10491066.

14. Ford, N., Wood, F. and Walsh, C. (1994) Cognitive Styles and Searching. Online \& CD-ROM Review, 18(2), 79-86.

15. Garrand, T. (1997). Writing for Multimedia: Entertainment, Education, Training, Advertising and the World Wide Web, Focal Press, Boston.

16. Garrett, B. M. and Callear, D. (2001) The value of intelligent multimedia simulation for teaching clinical decision-making skills. Nurse Education Today. 21(5), 382-390.

17. Ghinea, G. and Chen, S. Y. (2003) The Impact of cognitive styles on perceptual distributed multimedia quality. British Journal of Educational Technology. 34(4), 393-406.

18. Ghinea, G. and Thomas, J.P. (1998) QoS Impact on User Perception and Understanding of Multimedia Video Clips. Proceedings of ACM Multimedia ' 98.
19. Gill, A. J. (2003) Personality and Language: The projection and perception of personality in computermediated communication, Unpublished Ph.D. Dissertation, University of Edinburgh, UK.

20. Gross, R. D. (1994). Psychology - The Science of Mind and Behaviour, Hodder \& Stoughton, London.

21. Hapeshi, K. and Jones, D. (1992). Interactive Multimedia for Instruction: A Cognitive Analysis of the Role of Audition and Vision. International Journal of Human-Computer Interaction. 4(1), 79-99.

22. Huang, A.H. (2003) Effects of multimedia on document browsing and navigation: an exploratory empirical investigation. International Journal of Information and Management. 41, 189-198.

23. Lee, C. H. (1994) The Effects of Auditory Cues in Interactive Multimedia and Cognitive Style on Reading Skills of Third Graders, Unpublished Ed.D. Dissertation, University of Pittsburgh, USA.

24. Lewalter, D. (2003) Cognitive strategies for learning from static and dynamic visuals, Learning and Instruction. 13, 177-189.

25. Lowe, R.K. (2003) Animation and learning: selective processing of information in dynamic graphics, Learning and Instruction. 13, 157-176.

26. Mayer, R.E. (2003) The promise of multimedia learning: using the same instructional design methods across different media. Learning and Instruction. 13, 125-139.

27. Oberlander, J. and Gill, A.J. (2004) Individual differences and implicit language: Personality, partsof-speech and pervasiveness. In Proceedings of the 26th Annual Conference of the Cognitive Science Society, 1035-1040. Chicago, USA August 5-7, 2004.

28. Reeves, B. and Nass, C. (2000) Perceptual user interfaces: perceptual bandwidth. Communications of the ACM. 23(3), 65-70.

29. Reips, U.-D. (2000). The web experiment method: Advantages, disadvantages, and solutions. In Birnbaum, M. H., (Ed.), Psychological Experiments on the Internet, 89-117. Academic Press, San Diego.

30. Riding, R. J. (1991) Cognitive Styles Analysis, Birmingham: Learning and Training Technology.

31. Riding, R.J. and Grimley, M. (1999) Cognitive style and learning from multimedia materials in 11 year old children. British Journal of Educational Technology. 30(2), 43-56.

32. Riding, R.J. and Rayner, S.G. (1998) Cognitive Styles and Learning Strategies, London: David Fulton Publisher.

33. Schnotz, W. and Lowe, R. (2003) External and internal representations in multimedia learning. Learning and Instruction. 13(2), 117-123

34. Steinmetz, R. (1996) Human Perception of Jitter and Media Synchronisation. IEEE Journal on Selected Areas in Communications. 14(1), 61-72.

35. Stephen, P. and Hornby, S. (1997) Simple Statistics for Library and Information Professionals. London: Library Association.

36. Watson, A. and Sasse, M.A. (1997) Multimedia Conferencing via Multicast: Determining the Quality of Service Required by the End User. Proceedings of 
AVSPN '97 - International Workshop on AudioVisual Services over Packet Networks, Aberdeen, U.K.

37. Weller, H. G., Repman, J., and Rooze, G. E. (1994) The Relationship of learning, behavior, and cognitive styles in hypermedia-based instruction: Implications for design of HBI. Computers in the Schools. 10, 401420 .

38. Wijesekera, D., Srivastava, J., Nerode, A., and Foresti, M. (1999) Experimental Evaluation of Loss Perception in Continuous Media. Multimedia Systems. 7(6), 486-499.

39. Wilson, G. M. and Sasse, M. A. (2000) Investigating the Impact of Audio Degradations on Users: Subjective vs. Objective Assessment Methods. Proceedings of OZCHI'2000, Sydney, Dec. 2000.
40. Witkin, H. A., Oltman, P. K., Raskin, E., and Karp, S. A. (1971) A Manual For The Group Embedded Figures Test. Palo Alto, CA: Consulting Psychologists Press.

41. Witkin, H. A., Moore, C. A., Goodenough, D. R., \& Cox, P. W. (1977). Field-dependent and field independent cognitive styles and their educational implications. Review of Educational Research, 47( 1), $1-64$.

42. Xia, W. and Lee, G. (2000) The influence of persuasion, training and experience on user perceptions and acceptance of IT innovation. Proceedings of the 21st International Conference on Information Systems, Brisbane, Australia. 
